\title{
Effectiveness of the Group-Based Positive Parenting Program with Japanese Families Raising a Child with Developmental Disabilities: A Longitudinal Study
}

Rie Wakimizu ${ }^{1 *}$, Hiroshi Fujioka², Atsushi lejima ${ }^{3}$ and Shinya Miyamoto ${ }^{4}$

${ }^{1}$ Department of Child Health Care Nursing, Division of Health Innovation and Nursing, Faculty of Medicine, University of Tsukuba, Japan

${ }^{2}$ Department of Nursing, Tsukuba International University, Japan

${ }^{3}$ Department of Pediatric, Ibaraki Disabled Children's Hospital, Japan

${ }^{4}$ Department of Developmental Behavioral Pediatrics, Institute of Disability Sciences, Graduate School of Comprehensive Human Sciences, University of Tsukuba, Japan

\begin{abstract}
This study aims to investigate the effectiveness of a group-based family intervention program known as the Positive Parenting Program (Triple P) with families raising a child with developmental disabilities in Japan. Reductions in children's behavioral problems, changes in dysfunctional parenting practices, and promoting effects on family empowerment were examined. Participants $(\mathrm{N}=54)$ were recruited from mothers visiting two hospitals and two parental groups in the Tokyo metropolitan area. They were assessed in terms of child behavior (Strengths and Difficulties Questionnaire), parenting style (Parenting Scale), parenting adjustment (Depression Anxiety Stress Scale and Parenting Experience Survey), and family empowerment (Family Empowerment Scale). All outcomes were measured repeatedly from pre-intervention to six months after intervention. The Strengths and Difficulties Questionnaire scores for three sub scales emotional symptoms conduct problems, and difficult behavior indicated a significant intervention effect in the children. Moreover, the Parenting Scale scores for all subscales, the Depression Anxiety Stress Scale scores for all subscales, and the Parenting Experience Survey scores for the subscales except for the fulfilling subscale, the perception of agreement with partner regarding child discipline subscale, and the perception of support received from partner subscale, indicated a significant intervention effect in the caregivers. In addition, the Family Empowerment Scale scores for all subscales indicated a significant intervention effect in the family. These results provided promising evidence for the group intervention based Triple Pas an effective intervention program for Japanese families raising preschool and school-aged children with developmental disabilities.
\end{abstract}

Keywords: Developmental disability; Child rearing; Parent training; Family empowerment

\section{Introduction}

The number of children with Developmental Disabilities (DDs) is steadily increasing in Japan [1]. The results of the "National Survey on School Children with Special Educational Needs in a Regular Class" reported that $6.5 \%$ of all children have "mild DDs" that cause a significant difficulty in learning and behavior without mental retardation. A little fewer than $40 \%$ of these children have not received any special support. Furthermore, in-school committees should discuss support measures, but their work has been inadequate in ensuring that parents and family members can manage behavior correction and program development for problem behavior [2].

In addition, as shown by the recent establishment of the Act on Support for Persons with Developmental Disorders, as well as the enforcement of special-needs education [3], the supportive environment for DDs has improved, with a better understanding of disability in Japanese society. In Japan, DDs is defined as "autism, Asperger syndrome, and other pervasive developmental disorders, learning disorder, attention-deficit/hyperactivity disorder, and similar higher cerebral dysfunctions that usually present symptoms at an early age." The qualitative definition is described as "a disorder that typically causes behavior, communication, and social adjustment problems during the development process" [4].

Children with DDs often cause (or are involved in) problems (e.g. trouble with friends, isolation from other children, fall behind others) in the community or school one or more times [5]. Parents of a child with DDs need to deal with problems caused by the child and experience more stress associated with child rearing than other parents $[6,7]$. The pressure of childrearing felt by these parents is strong $[8,9]$. The presence of a child with DDs can also be a source of stress for the kin and siblings $[8,10]$. Although the large majority of parents adjust to raising these children, some fail to adapt; the latter are said to be potential child abusers [9]. Karst and Van Hecke [8] has reported that raising a child with DDs can lead to higher stress in the family.

In raising children with DDs, it is important to empower families in various ways such as through collaboration within the family, among several families, and between the family and the specialists or local government [11]. Empowerment refers to the conditions under which, or the attitude or action with which, individuals can achieve their goals through interactions with other people $[12,13]$. "Family empowerment" is an important indicator in other countries for both children with DDs and their families [14-16].

The Positive Parenting Program (Triple P) is a form of behavioral family interaction based on social learning principles; it was designed as a comprehensive, multi-level, prevention-oriented system of parenting support aimed at preventing and reducing childhood emotional and behavioral problems by promoting parental skills, knowledge, and confidence $[17,18]$. It is also based on research in child and family

*Corresponding author: Rie Wakimizu, Department of Child Health Care Nursing, Division of Health Innovation and Nursing, Faculty of Medicine, University of Tsukuba, 1-1-1, Tennodai, Tsukuba-city, 305-8575, Japan, Tel/Fax: +81-29-8533427; E-mail: riewaki@md.tsukuba.ac.jp

Received January 07, 2014; Accepted February 12, 2014; Published February 17,2014

Citation: Wakimizu R, Fujioka H, lejima A, Miyamoto S (2014) Effectiveness of the Group-Based Positive Parenting Program with Japanese Families Raising a Child with Developmental Disabilities: A Longitudinal Study. J Psychol Abnorm Child 3 : 113. doi:10.4172/2329-9525.1000113

Copyright: (c) 2014 Wakimizu R, et al. This is an open-access article distributed under the terms of the Creative Commons Attribution License, which permits unrestricted use, distribution, and reproduction in any medium, provided the original author and source are credited. 
behavior therapy and applied behavior analysis, focusing on antecedents of problem behavior and designing positive engaging environments for children. It draws on social information-processing models and targets parental cognitions, including attributions, expectancies, and beliefs. It is also based on research in developmental psychopathology and targets risk factors such as poor parent management practices, family discord, and parental distress. Therefore, it adopts a public health perspective and recognizes the role of the broader ecological context for human development $[19,20]$.

The Triple $\mathrm{P}$ advocates five core principles of positive parenting that address factors associated with positive developmental outcomes in children: (1) providing a safe and engaging environment, (2) providing a positive learning environment, (3) using assertive discipline, (4) having realistic expectations, and (5) taking care of oneself as a parent. Within the program, parents are taught a range of parenting strategies to enhance the quality of parent-child relationships, encourage desirable behavior, teach new skills, and manage misbehavior. Triple $\mathrm{P}$ emphasizes the development of parent self-regulation, which includes self-sufficiency, parental self-efficacy, self-management, and personal agency [17].

The Triple P comprises five levels of intervention, with group-based interventions (Group TripleP) included in level 4; this intervention is conducted for groups consisting of 10-12 parents. Results of various meta-analyses indicate that Group Triple P is effective in reducing child behavior problems and improving parenting outcomes such as parental stress, parental conflict, and dysfunctional discipline styles [21-23]. In East Asian cultures, Group Triple P has also been found to be effective with Hong Kong Chinese families [24,25] and Japanese families [26-28]. Group Triple P is thought to be quite robust across different cultures, despite cultural differences [26]. Additionally, Group Triple P emphasizes self-regulation, that is, parents form their own goals, which are informed by their culture rather than by simply adopting Western ideas about raising children. Therefore, it is likely that Japanese individuals in Japan can incorporate the ideas inherent in Group Triple P and adjust it in "Japanese" ways to improve their parenting practice and adjustment. This, in turn, will reduce children's behavioral problems and enhance family empowerment.

Although Group Triple P has been found to be effective with Japanese families [26-28], it is not clear whether this version is effective for Japanese families raising children with DDs. As noted above, parents raising children with DDs reported more child behavior problems, more stress, less well-being, and more social support than parents of children without DDs [7]. To promote the family empowerment process, provision of information on child rearing in local care resources has also been found to be essential for families raising children with DDs [29]. Therefore, we try to provide an effective support program based Group Triple P for children with DDs and their families to alleviate the family stress in child rearing in Japan.

The present study is the first attempt to investigate the effectiveness of a group-based Triple $\mathrm{P}$ intervention in reducing behavioral problems in children, changing dysfunctional parenting practices, and enhancing the status of empowerment of families raising a preschool or schoolaged child with DDs, using a longitudinal study design within a community setting in Japan.

\section{Methods}

\section{Recruitment of participants}

Participants of the study were the principal caregivers of the children. To include subjects with a variety of backgrounds, participants were recruited from the visitors of three urban and suburban hospitals for children with DDs in Ibaraki and two parents' associations of handicapped children in Tokyo. Caregivers of children aged 2-12 years with DDs were eligible, just followed the code of Triple P Japan. All children were assessed by pediatricians, who provided reports of their confirmed diagnoses before the intervention program.

\section{Procedures}

Caregivers decided whether to participate in the study after receiving an oral explanation of the study from a physician or researcher, along with a written document. Those who agreed to participate took the first self-administered questionnaire (pre-intervention questionnaire) home and, upon completion, brought it to the first session of group intervention. After the last session of the intervention, a set of questionnaires (post-intervention questionnaire) was mailed to each participant. Additionally, participants were asked to complete the questionnaires at 3 and 6 months after program completion (3months after-intervention questionnaire and 6 months after-intervention questionnaire). All questionnaires after the intervention were mailed back to the researcher from each participant in a sealed envelope. Those who did not send back the questionnaires before the due date were reminded by telephone and e-mail until they submitted their questionnaires.

\section{Intervention}

Group intervention was held five times at three Tokyo metropolitan areas from March 2011 to April 2013. In general, Group Triple P comprises eight sessions over an 8 week period, and it is ideally conducted in groups of 10-12 parents. Since it is extremely difficult to persuade Japanese fathers to participate in such programs, we focused primarily on mothers. The eight sessions areas follows: one group session geared toward providing an over view of the program and establishing a rapport with in the group (2heach), three group sessions in which parent training is conducted (2heach), three followup consultations by telephone (15-30 min each), and a final group session. The integrated telephone consultations provide additional support to mothers while they practice what they have learned in the group session, while following self-regulation theory. The parenttraining component involves discussions of 17 core child-management strategies designed to help parents promote their children's competence and development (e.g., praise for good behavior, creating engaging activities, and imparting incidental teaching) and to manage $\mathrm{m}$ is behavior (e.g., by setting rules, providing clear instructions, communicating the consequences of an action, and enforcing quiet time). In addition, participants are introduced to a "planned activities routine" to generalize what they have learned and help them strengthen their parenting skills. Active training methods such as modeling, rehearsal, practice, feedback, and goal setting are used throughout the program to teach specific parenting skills. In the final group session, participants share the knowledge and insights they acquired during the program, set future goals, and create plans to achieve those goals; the session also provides an opportunity for participants to celebrate the completion of the program.

\section{Data collection}

Data were collected from February 2011 to November 2013. The following outcomes were assessed by each measure. The measures consisted of a set of questionnaires to be completed by all participants four times, one time before and three times after the intervention. Participants were asked to complete these questionnaires again at 6 months after program completion. 
Child behavior: The Strengths and Difficulties Questionnaire (SDQ) developed by Goodman [30] was used in this study. The SDQ is a 25-item measure of parents' perceptions of prosocial and difficult behaviors in their child. The participants of this study rated the each 25 items on a scale of 0 to 2 . The SDQ consists of five subscales (emotional symptoms, conduct problems, hyperactivity, peer problems, and prosocial behavior), and the score for each subscale (range: $0-10$ ) is computed by summing the scores for the five items therein. And the difficult behavior score in the SDQ (range: 0-40) is calculated as the sum of the scores obtained for the emotional symptoms, conduct problems, hyperactivity, and peer problems subscales. It is designed to assess the frequency of positive and negative behaviors in children. The SDQ in Japanese has been validated as having adequate internal reliability (emotional symptoms, 0.61; conduct problems, 0.51; hyperactivity, 0.75 ; peer problems, 0.52 ; and prosocial behavior, 0.69 ) [31]. The difficult behavior score has also been found to have adequate internal reliability $(\mathrm{r}=0.76)$ and good test-retest reliability $(\mathrm{r}=0.85)$ [32].

Parenting style: The Parenting Scale (PS) developed by Arnold et al. [33] was used in this study. The PS is a 30 -item questionnaire that measures dysfunctional discipline styles in parents; it yields a total score based on three factors: (1) laxness (permissive discipline), (2) overreactivity (authoritarian discipline, displays of anger and irritability), and (3) verbosity (overly long reprimands or reliance on talking to impart discipline). The participants of this study rated the each 30 items on a scale of 1 to 7 . The higher score shows the more dysfunctional parenting. Total score (range: 1-7) is divided the sum of the score of all items by 30 . In the same way, scores of each factor is divided the sum of the score of all items in a factor by the number of items. The scale shows adequate internal consistency with respect to the total $(a=0.84)$, laxness $(\mathrm{a}=0.83)$, and over-reactivity $(\mathrm{a}=0.82)$, but only modest internal consistency with respect to verbosity $(\mathrm{a}=0.63)$. It also has good testretest reliability $(\mathrm{r}=0.84,0.83,0.82$, and 0.79 , respectively).

Parenting adjustment: The Depression-Anxiety-Stress Scale (DASS) developed by Lovibond and Lovibond [34] was used in this study. The DASS is a 42 -item questionnaire designed to assess symptoms of depression, anxiety, and stress in adults. The participants of this study rated the each 42 items on a scale of 0 to 3 . The higher score shows the higher depression-anxiety-stress state in parenting. The score for each subscale of depression, anxiety, and stress, is computed by summing the scores for the 14 items therein. And the total score in the DASS (range: 0-126) is calculated as the sum of the scores obtained for the depression, anxiety, and stress subscales. All three of the DASS subscales have been demonstrated to have high reliability (depression, $\mathrm{a}=0.91$; anxiety, $\mathrm{a}=0.84$; and stress, $\mathrm{a}=0.90$ ).

The Parenting Experience Survey (PES) developed by Turner et al. [35] was used in this study. In the PES, parents are asked to rate the following on a scale of 1 to 5 or $0-6:(1)$ the perceived level of difficult behavior in their child, (2) their subjective perceptions of their parenting role (e.g., how rewarding, demanding, and stressful they find parenting to be), (3) their confidence level and level of support they receive as a parent, (4) (for two-parent families) the extent of agreement between partners over child discipline and the level of support they receive from their partner in their role as parent, and (5) their degree of happiness with the relationship with their partner.

Family empowerment: The Family Empowerment Scale (FES) developed by Koren et al. [11] was used in this study. The FES is a 34item questionnaire designed to assess the empowerment of families taking care of children with emotional, behavioral, and developmental disorders. The participants of this study rated the each 34 items on a scale of 1 to 5. The FES consists of three subscales (family, service system, community/political), and the score for each subscale is computed by summing the scores for the items therein. The Family Empowerment Score in the FES (range: 34-170) is calculated as the sum of the scores obtained for the family, service system, and community/ political subscales (range: 12-60, 12-60, 10-50, respectively). The FES has been used in about 30 or more studies all over the world as a tool for measuring the empowerment of families taking care of children with DDs [36]. The FES in Japanese has been validated as having adequate internal consistency (family, 0.87; service system, 0.86; community/ political, 0.81) [37]. The total FES score has also been found to have adequate internal reliability $(\mathrm{r}=0.92)$ and good test-retest reliability $(\mathrm{r}=0.88)$ [38].

Client satisfaction: The Client Satisfaction Questionnaire (CSQ) developed by Turner et al. [39] was used in this study. The 13-item CSQ addresses quality of service provided, that is, how well the program met the parents' needs, increased parental skills, and decreased child behavior problems. Higher scores indicate higher satisfaction. The scale has high internal consistency (alpha $=.96)$, and has an item-total correlation of .66 and interim correlations of .30-.87 [40]. This was administered only post-intervention. Participants rated their degree of satisfaction with the service on a 7-point scale and a total score was calculated by summing up the scores.

Demographic information: Participants also provided basic demographic information on issues including gender, age, and disability of target child, education and occupation of parents, as well as family type, family income, marital status, and relationship of participant to the target child.

\section{Data analysis}

A one-factor repeated-measures ANOVA was used to analyze association between intervention and changes in outcome scores, and Bonferroni correction was applied to adjust for multiple comparisons. We showed the Effect Sizes (ES) in addition to the F and p- values for all the ANOVA analyses. According to Cohen's guidelines [41], we considered the ES-values (Small: 0.01/Medium: 0.059/Large: 0.138). And P-values of less than 0.05 were considered significant in the present study. All data analyses were conducted using SPSS for Windows, version20.0J, a Statistical Package for the Social Sciences (SPSS Japan Inc., Tokyo, Japan).

\section{Ethical consideration}

There searchers stated and observed the verbal and written agreements described below to candidate participants when requesting cooperation: (i) subject's cooperation in the survey is fully based on voluntary will, (ii) no disadvantage will occur to the medical care or treatment of the child even if the care giver does not cooperate in the study, (iii) consent to study cooperation can be withdrawn at any time during the study, and (iv) the privacy of subjects is strictly protected when the study results are published. The study was conducted with the approval of the Medical Ethics Review Board of the University of Tsukuba (approvalno.23-268).

\section{Results}

There were 55 participants in the study. One participant dropped out from the program due to family reasons. No participant failed to complete the pre- or post-intervention data throughout the 4 time points and at 6 months after intervention. 
Citation: Wakimizu R, Fujioka H, lejima A, Miyamoto S (2014) Effectiveness of the Group-Based Positive Parenting Program with Japanese Families Raising a Child with Developmental Disabilities: A Longitudinal Study. J Psychol Abnorm Child 3: 113. doi:10.4172/2329-9525.1000113

Page 4 of 9

\section{Summary of subjects}

Table 1 shows the characteristics of caregivers, families, and children. In age demographics, $22.2 \%$ of caregivers were in their

\begin{tabular}{|c|c|c|c|c|}
\hline \multicolumn{5}{|c|}{$(\mathrm{N}=54)$} \\
\hline & & & n/mean $\pm S D$ & $\begin{array}{l}\% / \\
\text { range }\end{array}$ \\
\hline \multicolumn{5}{|c|}{ Caregiver } \\
\hline & Gender & Female & 53 & $98.1 \%$ \\
\hline & Age & & $41.3 \pm 4.7$ & $34-52$ \\
\hline & Relationship & Mother & 53 & $98.1 \%$ \\
\hline & & Father & 1 & $1.9 \%$ \\
\hline & Employment status & Housewife & 27 & $50.0 \%$ \\
\hline & & Regular employee & 6 & $11.1 \%$ \\
\hline & & Part-timer & 19 & $35.2 \%$ \\
\hline & & Self-employed & 2 & $3.7 \%$ \\
\hline & Education level & High school & 12 & $22.2 \%$ \\
\hline & & Junior college & 9 & $16.7 \%$ \\
\hline & & $\begin{array}{l}\text { Professional } \\
\text { school }\end{array}$ & 12 & $22.2 \%$ \\
\hline & & College & 19 & $35.2 \%$ \\
\hline & & Graduate school & 2 & $3.7 \%$ \\
\hline & $\begin{array}{l}\text { People who help with child- } \\
\text { rearing }\end{array}$ & Yes & 51 & $94.4 \%$ \\
\hline & & No & 3 & $5.6 \%$ \\
\hline & $\begin{array}{l}\text { Use of service due to mental } \\
\text { issues }\end{array}$ & Psychiatrist & 10 & $18.5 \%$ \\
\hline & (multiple answers allowed) & $\begin{array}{l}\text { Clinical } \\
\text { psychologist }\end{array}$ & 4 & $7.4 \%$ \\
\hline & & Counselor & 4 & $7.4 \%$ \\
\hline & & Social worker & 2 & $3.7 \%$ \\
\hline \multicolumn{5}{|c|}{ Family } \\
\hline & Length of residence (year) & & $6.5 \pm 4.4$ & $0.3-20$ \\
\hline & Number of family members & & $3.9 \pm 0.9$ & $2-6$ \\
\hline & Number of children & 1 & 22 & $40.7 \%$ \\
\hline & & 2 & 23 & $42.6 \%$ \\
\hline & & 3 & 7 & $13.0 \%$ \\
\hline & & 4 & 2 & $3.7 \%$ \\
\hline & Annual household income & $<3$ & 3 & $5.6 \%$ \\
\hline & (in millions of yen) & $3-5$ & 12 & $22.2 \%$ \\
\hline & & $5-7$ & 18 & $33.3 \%$ \\
\hline & & $7-10$ & 13 & $24.1 \%$ \\
\hline & & $10-13$ & 6 & $11.1 \%$ \\
\hline & & $>13$ & 2 & $3.7 \%$ \\
\hline \multicolumn{5}{|c|}{ Child } \\
\hline & Gender & Male & 42 & $77.8 \%$ \\
\hline & Age (years) & & $7.4 \pm 2.7$ & $2-14$ \\
\hline & $\begin{array}{l}\text { Diagnosis (multiple answers } \\
\text { allowed) }\end{array}$ & $\mathrm{ADHD}^{1)}$ & 34 & $63.0 \%$ \\
\hline & & $\mathrm{PDD}^{2)}$ & 26 & $48.1 \%$ \\
\hline & & Autism & 11 & $20.4 \%$ \\
\hline & & $\mathrm{AS}^{3)}$ & 5 & $9.3 \%$ \\
\hline & & $\mathrm{LD}^{4)}$ & 5 & $9.3 \%$ \\
\hline & & $\begin{array}{l}\text { MR }{ }^{5} \text { (including } \\
\text { mild MR }\end{array}$ & 3 & $5.6 \%$ \\
\hline & & Epilepsy & 3 & $5.6 \%$ \\
\hline & & $\mathrm{DCD}^{6)}$ & 3 & $5.6 \%$ \\
\hline & & Anxiety disorder & 2 & $3.7 \%$ \\
\hline & & $\begin{array}{l}\text { Adjustment } \\
\text { disorder (truant) }\end{array}$ & 2 & $3.7 \%$ \\
\hline
\end{tabular}

\begin{tabular}{|l|l|r|r|}
\hline & Tic disorder & 2 & $3.7 \%$ \\
\hline & Nocturnal enuresis & 1 & $1.9 \%$ \\
\hline & $\begin{array}{l}\text { Obsessive- } \\
\text { compulsive } \\
\text { disorder }\end{array}$ & 1 & $1.9 \%$ \\
\hline & Unknown & 5 & $9.3 \%$ \\
\hline Medication & Yes & 42 & $77.8 \%$ \\
\hline & No & 12 & $22.2 \%$ \\
\hline Disability certificate & Yes & 9 & $16.7 \%$ \\
\hline & No & 45 & $83.3 \%$ \\
\hline Attendance at (pre)school & Regular class & 34 & $63.0 \%$ \\
\hline & Support class & 14 & $25.9 \%$ \\
\hline & Special-needs & 3 & $5.6 \%$ \\
\hline & class & 3 & $5.6 \%$ \\
\hline & Private nursery & 10 & $18.5 \%$ \\
\hline & Yes & 44 & $81.5 \%$ \\
\hline Use of Tsukyu & No & \\
\hline & & & \\
\hline 1) ADHD: Attention Deficit / Hyperactivity Disorder & & \\
\hline 2) PDD: Pervasive Developmental Disorders & & \\
\hline 3) AS: Asperger syndrome & & \\
\hline 4) LD: Learning Disorders & & \\
\hline 5) MR: Mental Retardation & & \\
\hline 6) DCD: Developmental Coordination Disorder & & \\
\hline
\end{tabular}

Table 1: Characteristics of subjects.

$30 \mathrm{~s}, 74.1 \%$ were in their $40 \mathrm{~s}$, and $3.7 \%$ were in their $50 \mathrm{~s}$. The rate of caregivers who answered that they had someone help them raise their child was $94.4 \%$. Their husbands or grandparents were listed as the helper. However, the use of local services, including "consultation for therapeutic education", "temporary support in the daytime", "support available outside", and "teaching language, therapeutic education and training for the child", was low at $32.0 \%$. Children were $7.4 \pm 2.7$ years old (mean \pm SD), and boys accounted for $77.8 \%$ of the total. Medication was taken by $77.8 \%$ of children, and $16.7 \%$ had an intellectual disability certificate. With regard to attendance at preschools and schools, $63.0 \%$ of children attended a regular class, which exceeded $25.9 \%$ of those who attended a support class and $5.6 \%$ of those who attended a special-needs class. The $18.5 \%$ of children used Tsukyu (school system that provides special education as needed with children with DDs attending a regular class) because of limited availability.

\section{Follow-up results and intervention effects}

Table 2 presents the means and standard deviations of each dependent measure before and after the intervention, along with the $\mathrm{F}$ statistics and significance values yielded by the repeated-measures ANOVA (controlled for baseline scores), all of which show the effect of the intervention.

Child behavior: The repeated-measures ANOVA revealed a significant intervention effect on the SDQ scores for the emotional symptoms subscale $(\mathrm{F}=4.77, \mathrm{p}=0.003, \mathrm{ES}=0.081)$, the conduct problems subscale $(\mathrm{F}=2.79, \mathrm{p}=0.042, \mathrm{ES}=0.049)$, and the difficult behavior subscale $(\mathrm{F}=10.39, \mathrm{p}<0.001, \mathrm{ES}=0.108)$. The level of emotional symptoms and the conduct problems were significantly lower in the 3 months after the intervention period. The level of difficult behavior was significantly lower after the intervention until the 6-month period. In addition, the mean score for the difficult behavior subscales was within the clinical range [31] before the intervention, but outside the clinical range after intervention. There were no significant effects of the child's gender, type of educational setting the child was receiving, and residence area on the SDQ scale. 
Citation: Wakimizu R, Fujioka H, lejima A, Miyamoto S (2014) Effectiveness of the Group-Based Positive Parenting Program with Japanese Families Raising a Child with Developmental Disabilities: A Longitudinal Study. J Psychol Abnorm Child 3: 113. doi:10.4172/2329-9525.1000113

Page 5 of 9

\begin{tabular}{|c|c|c|c|c|c|c|c|c|}
\hline \multirow{2}{*}{\begin{tabular}{|l|}
$(\mathbf{N}=54)$ \\
Scale
\end{tabular}} & \multirow[b]{2}{*}{ Score range } & \multirow{2}{*}{$\begin{array}{c}\text { Time } \\
\text { Pre Mean } \\
\text { (SD) }\end{array}$} & \multirow[b]{2}{*}{$\begin{array}{l}\text { Post Mean } \\
\text { (SD) }\end{array}$} & \multirow[b]{2}{*}{$\begin{array}{c}3 \text { months after } \\
\text { Mean (SD) }\end{array}$} & \multirow[b]{2}{*}{$\begin{array}{c}6 \text { months after } \\
\text { Mean (SD) }\end{array}$} & \multicolumn{3}{|c|}{ Effect (time effect) } \\
\hline & & & & & & $\mathrm{F}^{\pi}$ & $p$-value & effect sizes \\
\hline \multicolumn{9}{|l|}{ SDQ } \\
\hline Emotional symptoms & $0-10$ & $2.98(2.32)$ & $1.98(1.65)$ & $1.87(1.67)^{*}$ & $2.03(1.97)$ & 4.77 & 0.003 & 0.081 \\
\hline Conduct problems & $0-10$ & $3.46(2.19)$ & $2.89(1.83)$ & $2.47(1.87)^{*}$ & $2.79(2.12)$ & 2.79 & 0.042 & 0.049 \\
\hline Hyperactivity & $0-10$ & $6.00(2.65)$ & $5.11(2.29)$ & $5.04(2.33)$ & $5.34(2.17)$ & 2.05 & 0.109 & 0.037 \\
\hline Peer problems & $0-10$ & $4.94(2.45)$ & $4.19(2.19)$ & $3.84(2.38)$ & $4.39(2.70)$ & 2.26 & 0.084 & 0.040 \\
\hline Difficult behavior & $0-40$ & $17.38(5.89)$ & $14.17(5.10)^{* *}$ & $13.22(6.00)^{* * *}$ & $14.55(5.95)^{*}$ & 6.52 & $<0.001$ & 0.108 \\
\hline Prosocial behavior & $0-10$ & $4.07(2.68)$ & $4.50(2.65)$ & $4.87(2.63)$ & $4.89(2.36)$ & 1.31 & 0.272 & 0.024 \\
\hline \multicolumn{9}{|l|}{ PS } \\
\hline Laxness & $1-7$ & $2.79(0.49)$ & $2.29(0.64)^{* * *}$ & $2.22(0.72)^{+* t+}$ & $2.23(0.64)^{*+*}$ & 12.16 & $<0.001$ & 0.184 \\
\hline Over-reactivity & $1-7$ & $3.78(1.17)$ & $2.75(1.21)^{* * *}$ & $2.84(1.14)^{*+* t}$ & $3.12(1.19)^{\star}$ & 10.39 & $<0.001$ & 0.161 \\
\hline Verbosity & $1-7$ & $3.65(0.98)$ & $2.65(0.84)^{* * *}$ & $2.81(1.01)^{*+* t}$ & $2.91(0.96)^{* * *}$ & 13.63 & $<0.001$ & 0.202 \\
\hline Total & $1-7$ & $3.27(0.62)$ & $2.50(0.72)^{* * *}$ & $2.54(0.78)^{+* t *}$ & $2.63(0.70)^{*+*}$ & 17.08 & $<0.001$ & 0.240 \\
\hline \multicolumn{9}{|l|}{ DASS } \\
\hline Depression & $0-42$ & $8.25(8.33)$ & $4.72(5.84)^{\star}$ & $4.00(6.17)^{* *}$ & $6.58(7.32)$ & 4.23 & 0.007 & 0.100 \\
\hline Anxiety & $0-42$ & $5.51(5.00)$ & $3.74(3.57)$ & $3.29(5.05)^{*}$ & $5.50(5.53)$ & 3.61 & 0.022 & 0.065 \\
\hline Stress & $0-42$ & $12.45(7.43)$ & $7.96(5.95)$ & $7.51(6.72)$ & $11.39(8.82)$ & 5.52 & 0.001 & 0.127 \\
\hline Total & $0-126$ & $\begin{array}{c}26.22 \\
(18.33)\end{array}$ & $16.43(13.72)^{*}$ & $14.80(16.98)^{* *}$ & $22.97(20.06)$ & 5.21 & 0.002 & 0.121 \\
\hline \multicolumn{9}{|l|}{ PES } \\
\hline Parenting is difficult & $1-5$ & $3.38(0.98)$ & $2.60(0.90)^{* * *}$ & $2.51(0.83)^{+* t+}$ & $3.14(1.07)$ & 14.69 & $<0.001$ & 0.217 \\
\hline Parenting is rewarding & $1-5$ & $2.70(0.97)$ & $3.13(0.90)^{*}$ & $3.27(1.10)^{\star}$ & $2.81(1.14)$ & 4.34 & 0.006 & 0.076 \\
\hline Parenting is demanding & $1-5$ & $3.43(1.05)$ & $2.72(1.10)^{* *}$ & $2.93(1.16)$ & $3.27(1.11)$ & 5.78 & 0.001 & 0.098 \\
\hline Parenting is stressful & $1-5$ & $3.39(1.01)$ & $2.63(1.04)^{* * *}$ & $2.56(1.01)^{* *}$ & $3.16(1.13)$ & 8.28 & $<0.001$ & 0.135 \\
\hline Parenting is fulfilling & $1-5$ & $2.87(1.12)$ & $3.72(1.04)$ & $2.98(0.93)$ & $2.66(0.78)$ & 15.53 & $<0.001$ & 0.227 \\
\hline Parenting is depressive & $1-5$ & $3.22(1.21)$ & $2.41(1.11)^{+* *}$ & $2.42(1.02)^{* *}$ & $2.76(1.17)$ & 8.63 & $<0.001$ & 0.140 \\
\hline Confidence in parenting & $1-5$ & $2.24(0.90)$ & $2.96(0.72)^{*+*}$ & $2.78(0.84)^{* *}$ & $2.74(0.75)^{* *}$ & 9.91 & $<0.001$ & 0.157 \\
\hline Support for parenting & $1-5$ & $2.67(0.98)$ & $3.39(1.04)^{\star \star}$ & $3.11(0.82)^{*}$ & $2.82(1.07)$ & 6.61 & $<0.001$ & 0.111 \\
\hline $\begin{array}{l}\text { Agreement with partner regarding child discipline } \\
(n=51)\end{array}$ & $1-5$ & $2.86(0.99)$ & $3.08(1.06)$ & $3.14(0.89)$ & $3.03(1.00)$ & 1.07 & 0.366 & 0.021 \\
\hline Support received from partner $(n=51)$ & $1-5$ & $2.90(1.03)$ & $3.18(1.09)^{\star}$ & $3.19(1.18)$ & $3.05(1.18)$ & 1.09 & 0.356 & 0.021 \\
\hline Happiness with partner $(n=51)$ & $0-6$ & $3.12(1.25)$ & $3.32(1.24)$ & $3.62(1.23)$ & $3.03(1.13)$ & 3.37 & 0.020 & 0.063 \\
\hline \multicolumn{9}{|l|}{ FES } \\
\hline Family & $12-60$ & $37.55(6.22)$ & $42.07(7.12)^{*+*+}$ & $41.98(6.66)^{* *}$ & $40.11(7.09)^{\star}$ & 7.65 & $<0.001$ & 0.126 \\
\hline Service system & $12-60$ & $40.40(5.29)$ & $43.52(6.41)^{*+* t}$ & $43.29(7.49)$ & $43.55(7.20)$ & 4.10 & 0.008 & 0.072 \\
\hline Community/political & $10-50$ & $25.71(6.57)$ & $27.85(6.29)^{* *}$ & $27.96(7.08)$ & $28.08(6.38)$ & 2.09 & 0.104 & 0.038 \\
\hline Total & $34-170$ & $\begin{array}{l}103.65 \\
(15.45)\end{array}$ & $113.44(17.40)^{* * *}$ & $112.27(17.76)^{*}$ & $111.74(18.50)$ & 5.03 & 0.002 & 0.087 \\
\hline
\end{tabular}

ANOVA adjusted for the baseline score at each measurement time.

" $p<0.05,{ }^{* *} p<0.01,{ }^{* * *} p<0.001$,

"F statistic in repeated measures ANOVA adjusted for each baseline score.

ANOVA, analysis of variance; SD, standard deviation

Table 2: Results of repeated measures ANOVA on children, caregivers, and family outcomes.

Parenting style: The repeated-measures ANOVA indicated a strong significant intervention effect on all the subscales of the PS: the laxness $(\mathrm{F}=12.16, \mathrm{p}<0.001, \mathrm{ES}=0.184)$, the over-reactivity $(\mathrm{F}=10.39, \mathrm{p}<0.001$, $\mathrm{ES}=0.161)$, the verbosity $(\mathrm{F}=13.63, \mathrm{p}<0.001, \mathrm{ES}=0.202)$, and the total PS score $(\mathrm{F}=17.08, \mathrm{p}<0.001, \mathrm{ES}=0.240)$. The present study showed a significant reduction in the use of dysfunctional parenting strategies after intervention until the 6-month period. There were no significant effects of the child's gender, type of educational setting the child was receiving, and residence area on the PS scale.

Parenting adjustment: According to the repeated-measures ANOVA, the post-intervention mean score for the DASS depression subscale and the DASS total score were significantly reduced until 
Citation: Wakimizu R, Fujioka H, lejima A, Miyamoto S (2014) Effectiveness of the Group-Based Positive Parenting Program with Japanese Families Raising a Child with Developmental Disabilities: A Longitudinal Study. J Psychol Abnorm Child 3: 113. doi:10.4172/2329-9525.1000113

Page 6 of 9

3months after the intervention $(\mathrm{F}=4.23, \mathrm{p}=0.007, \mathrm{ES}=0.100 ; \mathrm{F}=5.21$, $\mathrm{p}=0.002$, $\mathrm{ES}=0.121$ ). Overall, the repeated-measures ANOVA revealed a significant intervention effect on each DASS subscale and total score. There were no significant effects of the child's gender, type of educational setting the child was receiving, and residence area on the DASS scale.

The repeated-measures ANOVA for the PES revealed a significant intervention effect on (1) the mothers' perceived level of difficult behavior in their child $(\mathrm{F}=14.69, \mathrm{p}<0.001, \mathrm{ES}=0.217)$; (2) their subjective perceptions of their parenting role (rewarding: $\mathrm{F}=4.34$, $\mathrm{p}=0.006, \mathrm{ES}=0.076$; demanding: $\mathrm{F}=5.78, \mathrm{p}=0.001, \mathrm{ES}=0.098$; stressful: $\mathrm{F}=8.28, \mathrm{p}<0.001, \mathrm{ES}=0.135$; fulfilling: $\mathrm{F}=15.53, \mathrm{p}<0.001, \mathrm{ES}=0.227$; depressive: $\mathrm{F}=8.63, \mathrm{p}<0.001, \mathrm{ES}=0.140$ ); (3) their confidence level in parenting $(\mathrm{F}=9.91, \mathrm{p}<0.001, \mathrm{ES}=0.157)$; (4) their perceived level of support in their parenting role $(\mathrm{F}=6.61, \mathrm{p}<0.001$, $\mathrm{ES}=0.111)$; and (5) their degree of happiness with the relationship with their partner $(\mathrm{F}=3.37, \mathrm{p}=0.020, \mathrm{ES}=0.063)$. However, the repeated-measures ANOVA did not demonstrate a significant intervention effect on the mothers' ratings, indicating (6) the extent of agreement between them and their respective partners regarding child discipline, as well as (7) the level of support they receive from their partners in their role as parent.

Family empowerment: The repeated-measures ANOVA indicated a significant intervention effect on two subscales of the FES: the family $(\mathrm{F}=7.65, \mathrm{p}<0.001, \mathrm{ES}=0.126)$ and the service system $(\mathrm{F}=4.10, \mathrm{p}=0.008$, $\mathrm{ES}=0.072)$, and the total FES score $(\mathrm{F}=5.03, \mathrm{p}=0.002, \mathrm{ES}=0.087)$. This study showed a significant enhancement in the family empowerment after intervention until the 3-month period, at least. There were no significant effects of the child's gender, type of educational setting the child was receiving, and residence area on the FES scale.

\section{Client satisfaction}

On the Client Satisfaction Questionnaire (CSQ), among the 54 participants who completed the program, a part from the question on improvement in relationship with spouse (mean=4.34), the mean scores of all other items were above 5 on the 7 -point scale (Table 3 ).

\section{Discussion}

This was the first study that investigated the effectiveness of group intervention based Group Triple P in reducing behavioral problems in children, changing dysfunctional parenting practices, and enhancing the status of empowerment of families raising a preschool or school aged child with DDs, using a longitudinal study design within a community setting in Japan. We think the participants of this study were a good representation of the population viewed from the annual household income because an average of that was 5,48 million of yen according to the announcement of Ministry of Health, Labour and Welfare in 2013 [42].

\section{Follow-up results and intervention effects}

Child behavior: The present study clearly showed the effectiveness of Group Triple P in reducing emotional symptoms, conduct problems, and difficult behaviors of children with DDs as measured with the SDQ. Compared with the previous study with parents of typically developing children in Japan $[25,26]$, the SDQ scores for the emotional symptoms, conduct problems, hyperactivity, peer problems, and difficult behavior subscales before starting the intervention (at baseline) were higher and the SDQ score for the prosocial behavior subscale before starting the intervention (at baseline) was lower in the subjects in this study (parents of children with DDs), which was as expected. According to the cutoff classification set (low-need, some-need, and high-need) based on "Standards for SDQ in Japan (data analysis of 2,899 children aged 4 to 12 years)"published by the Ministry of Health, Labour and Welfare in Japan [43], the baseline values of hyperactivity and peer problems of the subjects in this study were classified as some-need, while those with difficult behavior and prosocial behavior were classified as high-need.

Group Triple $\mathrm{P}$ is a direct and concrete parenting program. We were initially concerned about the efficacy of Group Triple P intervention in changing child behavior, as was the case in the previous study [26], because (1) the children participating in this study have conduct problems as their disability characteristics and (2) Group Triple P is not a program for child behavior correction, but for practical strategies to be used by the caregiver. However, contrary to the prediction, their conduct problems (emotional symptoms, conduct problems, and difficult behavior) evaluated by parents decreased, which was maintained for 3or 6 months. The level of difficult behavior was significantly lower after the intervention. In addition, the cut-off class was maintained at some-need and the scores were outside the clinical range; the scores were maintained for 6months. The difficult behavior subscale is a summed score of 4 items, including emotional symptoms, conduct problems, hyperactivity, and peer problems, but we also considered the idea that it was a multiplicative effect. Unfortunately, intervention was not effective in prosocial behavior, which remained

\begin{tabular}{|c|c|c|}
\hline$(\mathrm{N}=54)$ & & \\
\hline & mean & SD \\
\hline 1. How would you rate the quality of help you and your child received? $(7=$ Excellent, $1=$ poor $)$ & 5.94 & 1.00 \\
\hline 2. Did you receive the type of help you wanted from the programme? ( $7=$ Yes definitely, $1=$ No, definitely not $)$ & 5.91 & 0.90 \\
\hline 3. To what extent has the programme met your child's needs? $(7=$ Almost all have been met, $1=$ No needs have been met $)$ & 5.06 & 1.25 \\
\hline 4. To what extent has the programme met your needs? $(7=$ Almost all have been met, $1=$ No needs have been met $)$ & 5.40 & 1.25 \\
\hline 5. How satisfied were you with the amount of help you and your child received? $(7=$ Very satisfied, $1=$ Quite dissatisfied $)$ & 6.08 & 0.95 \\
\hline $\begin{array}{l}\text { 6. Has the programme helped you to deal more effectively with your child's behaviour? }(7=\text { Yes it has helped a great deal, } 1=\text { No it has made } \\
\text { things worse) }\end{array}$ & 5.45 & 1.27 \\
\hline $\begin{array}{l}\text { 7. Has the programme helped you to deal more effectively with problems that arise in your family? }(7=\text { Yes it has helped a great deal, } 1=\text { No it has } \\
\text { made things worse) }\end{array}$ & 5.17 & 1.14 \\
\hline 8. Do you think your relationship with your partner has been improved by the programme? $(7=$ Yes definitely, $1=$ No, definitely not $)$ & 4.34 & 1.45 \\
\hline 9. In an overall sense, how satisfied are you with the programme you and your child received? $(7=$ Very satisfied, $1=$ Quite dissatisfied $)$ & 5.96 & 1.05 \\
\hline 10. If you were to seek help again, would you come back to Triple $P$ ? $(7=$ Yes definitely, $1=$ No, definitely not $)$ & 5.33 & 1.19 \\
\hline 11. Has the programme helped you to develop skills that can be applied to other family members? $(7=$ Yes definitely, $1=$ No, definitely not $)$ & 5.02 & 1.37 \\
\hline 12. In your opinion, how is your child's behaviour at this point? ( 7 = Greatly improved, $1=$ Considerably worse $)$ & 5.51 & 0.74 \\
\hline 13. How would you describe your feelings at this point about your child's progress? $(7=$ Very satisfied, $1=$ Very dissatisfied $)$ & 5.44 & 1.18 \\
\hline
\end{tabular}

Table 3: Results of the Client Satisfaction Questionnaire items. 
within the clinical range. A more directly encouraging program, as well as Group Triple $\mathrm{P}$, is required by parents in order to improve children's prosocial behavior within the limited timeframe. However, this study result indicated that Group Triple P indirectly but effectively changed conduct problems of children of concern to caregivers.

Parenting style: The present study found that Group Triple P was able to bring about great improvements in parenting style among Japanese participants rearing children with DDs. The scores of child behavior at baseline was high, which indicated difficulty raising children, but the PS scores for all subscales at baseline were lower than those of the previous study in parents of typically developing children $[26,28]$, which was not expected. Parents of children with DDs in Japan may have some educational opportunities regarding management of their children in outpatient departments of child psychiatric or medical centers; therefore, they may be trained to some extent before starting the intervention. Sessions 2 and 3 of Group Triple P provide " 10 skills to establish good relationship with their children to promote their development" and "7 skills to handle behavior of difficult children", respectively [17]. Both sessions teach direct and concrete skills for caregivers. Overall, the intervention significantly decreases PS scores, and these effects last 6 months.

It is obvious that Group Triple $\mathrm{P}$ was effective in modifying parenting style of caregivers in this study. There were some reasons the intervention effect lasted 6months in PS. First, Group Triple P is a program focusing on 17 child-rearing skills, which is acceptable and easy to use for caregivers, and second, the contents of Group Triple P are described in one textbook, which may enable them to repeatedly read and recall it. For another reason, we thought that the Group Triple P allowed parents to self-regulate, choose which areas to target, and internalize the "lessons" to improve their parenting practice and adjustment because of the group-based intervention style was part of Japanese culture.

Parenting adjustment: The present study clearly showed the effectiveness of Group Triple P in reducing participant's depression, anxiety, and stress, as measured with the DASS and in decreasing the extent to which participants found parenting to be difficult, demanding, stressful, and depressive, and in improving the extent to which they found parenting to be rewarding and their confidence in their parenting skills.

Each subscale score and the sums of DASS at baseline were higher than those of the previous study in parents of typically developing children [26,34]. Recent studies reported that $32.2 \%$ of women in their 40s in Japan had some psychiatric symptoms and approximately $30 \%$ of them visited medical institutions for consultation $[44,45]$. The percentage of those using mental health services was quite higher in the subjects of this study (Table 1) compared with the above-mentioned reports. In Japan, mothers take their children with DDs to routine consultations, and doctors evaluate the state of not only children, but also their mothers, and refer the mothers to other specialists (psychiatrists, psychologists, counselors, etc.) if necessary. This may explain the above-mentioned high percentage of service use. This study result revealed that the intervention significantly decreased DASS scores, which lasted 3months. Parents of children with DDs are likely to worry about how to be involved with their children and to deal with their conduct problems $[7,46]$. It was assumed that the above applied to the majority of subjects in this study. Therefore, Group Triple P, which focuses on concrete child-rearing skills of caregivers, was suitable for them.

The results of the PES indicated that (1) the mothers' perceived level of difficult behavior in their child, at baseline, was higher than that in the previous study in parents of typically developing children [26], which was consistent with the result of 4.1.1; and (2) their subjective negative perceptions of their parenting role (like "low-rewarding" and "high-demanding", stressful, and depressive) at baseline were higher while their subjective positive perceptions at baseline were lower than those of the previous study. In addition, (3) their confidence level in parenting, (4) their perceived level of support in their parenting role, (5) their degree of happiness with the relationship with their partner, (6) the extent of agreement between them and their respective partners regarding child discipline, and (7) the level of support they receive from their partners in their role as parent at baseline were lower than those of the previous study [26]. In terms of parenting experience of child-rearing based on the above-mentioned findings, the caregivers in this study considered their parenting as negative tasks containing non-positive factors, lost their confidence, had problems with their partners, and felt isolation; in other words, they themselves needed help. The intervention effects associated with the outcomes of (1) to (5) and, in particular, (3), significantly increased, and this effect lasted for 6 months. The previous study reported that caregivers' confidence in child rearing significantly influenced the quality of child rearing and caregivers themselves, and their children and family members. It was significant that Group Triple P improved the outcome of (3) and the effect lasted a long time. In general, the outcomes of (1), (2), (4), and (5) lasted at least 3 months. Therefore, the caregivers in this study used the skills obtained in Group Triple P in the daily rearing of their children and showed various changes and obtained good results, which might change parenting perceptions.

However, the current study did not find Group Triple P to be effective in bringing about improvements in areas related to the parents' partner. This might be due to our study method, because Group Triple $\mathrm{P}$ was performed in the daytime on weekdays, the majority of the participants were mothers. In addition, Japan is unique in that fathers work hard from the morning to midnight and have no time for communication with and support for mothers about child rearing; this might have contributed to the result. Another reason the intervention effect may have been insufficient is that the purpose and contents of Group Triple P mainly focus on the relationship with children, not partners. Group Triple P should be targeted toward both the mother and father. However, since we carried out the Group Triple P sessions on weekdays, participation by working fathers was not possible. The findings of previous studies have suggested the association between paternal involvement and child functioning [47-49], which in turn suggests better out comes if fathers are involved in Group Triple P. Future studies should involve fathers and test the effectiveness of Group Triple P on scales related to both parents.

Family empowerment: This study was the first to measure family empowerment as the primary outcome of Group Triple P. The present study found Group Triple $\mathrm{P}$ to be effective in bringing about improvements in the empowerment of the family rearing children with DDs. Each subscale and sums of FES at baseline were more similar compared with the previous study in patients of children with DDs in Japan [37]. In fact, FES scores of caregivers of children with DDs in Japan were significantly lower than those of the study conducted overseas $[11,50]$. The intervention effects were demonstrated by 'family' and 'service system' subscale scores and sums of the scores. In particular, the subscale scores of family significantly increased by the intervention and the effects lasted 6months. Moreover, the subscale scores of service system were significantly greater immediately after the intervention, but did not last. As described above, the reason the longterm intervention effects were observed only in the subscale scores of 
Citation: Wakimizu R, Fujioka H, lejima A, Miyamoto S (2014) Effectiveness of the Group-Based Positive Parenting Program with Japanese Families Raising a Child with Developmental Disabilities: A Longitudinal Study. J Psychol Abnorm Child 3: 113. doi:10.4172/2329-9525.1000113

family might be that the purpose and contents of Group Triple P mainly focus on establishment of the relationship between caregivers and children at home. However, this study demonstrated the intervention effect of Group Triple P on family empowerment, overall.

\section{Client satisfaction}

Client satisfaction with the Group Triple P program in this study was evaluated after the intervention, and was higher than that in the previous studies $[39,40]$, suggesting that the program was easy to use for them. Sanders et al. [40] reported that all CSQ scores were greater in the enhanced group than in the standard group. In this study, all outcomes (child behavior, parenting style, parenting adjustment, and family empowerment) of Group Triple P were significantly greater, which might be related to their high satisfaction.

\section{Limitations of the present study and issues for the future}

The present study had some limitations. First, this study did not divide the subjects into an intervention group and a waitlist control group to compare outcomes, so we couldn't determine that the present intervention was better than time and maturation. Second, Group Triple $\mathrm{P}$ intervention was performed in the daytime on weekdays, so the majority of the participants were mothers. Third, although there are various types of DDs, the caregivers in this study were enrolled as "parents of children with developmental disability" without any detailed classification. Fourth, all the measures were reported by the caregivers, who wanted to believe that their children are improving and that they are fulfilling their training well.

It is necessary and desirable to carry out further studies that use a randomized design in order to assess the long-term effects of intervention and confirm the validity of the current study's results. Additionally, future trials would need to make provisions to allow the participation of family members, so the whole family can be consistent in applying the Triple P strategies. Finally, children with different DDs may have different needs and characteristics, and parents are likely to come from diverse backgrounds. Triple $\mathrm{P}$ facilitators need to be sensitive to the different needs of children and families and be flexible in delivery while retaining program fidelity [51]. Furthermore, the effects of intervention should be evaluated in terms of not only child outcomes but also parent and family factors that may have an influence on both the immediate and long-term effects of therapy or treatment $[8,52]$. Adding an observational piece, especially a blind observer would also be a key element.

Nevertheless, the current study recommends that Group Triple P intervention be employed widely in Japan as an effective improvement and relief program for the families who are raising preschool or schoolaged children with DDs.

\section{Acknowledgement}

This study was a research project funded by a grant from the Ministry of Education, Culture, Sports, Science and Technology, Japan (Grant-in Aid for Young Scientists (A), 2011-2013, No.23689089).

\section{References}

1. Japan League on Developmental Disabilities (2013) HattatsuShougaiHakusho. Akashi Shoten, Tokyo, Japan.

2. Ministry of Education, Culture, Sports, Science and Technology (2012) Report on national survey on school children with special educational needs in a regular class.

3. Ministry of Health, Labour and Welfare (2005) Establishment of the support law for people with developmental disorders.

4. Hiraiwa M (2009) A statement about children's mental and behavioral issue. J Child Health 68: 330 .
5. Takahashi S (2008) School maladjustment and problems of educational support for students with mild developmental disabilities: A survey of resource rooms for students with emotional disturbances in elementary and lower secondary schools in Tokyo. Jpn J Spec Educ 45: 527-541.

6. Anderson LS (2008) Predictors of parenting stress in a diverse sample of parents of early adolescents in high-risk communities. Nurs Res 57: 340-350.

7. Nachshen JS, Minnes P (2005) Empowerment in parents of school-aged children with and without developmental disabilities. J Intellect Disabil Res 49: 889-904.

8. Karst JS, Van Hecke AV (2012) Parent and family impact of autism spectrum disorders: a review and proposed model for intervention evaluation. Clin Child Fam Psychol Rev 15: 247-277.

9. Raina P, O'Donnell M, Schwellnus H, Rosenbaum P, King G, et al. (2004) Caregiving process and caregiver burden: conceptual models to guide research and practice. BMC Pediatr 4: 1.

10. Schuntermann $P$ (2007) The sibling experience: growing up with a child who has pervasive developmental disorder or mental retardation. Harv Rev Psychiatry 15: 93-108.

11. Koren PE, DeChillo N, Friesen BJ (1992) Measuring empowerment in families whose children have emotional disabilities: A brief questionnaire Rehab Psychol 37: 305-321.

12. Conger JA, Kanungo RN (1988) The empowerment process: Integrating theory and practice. Acad Manage Rev 13: 471-482.

13. Dunst C, Trivette C, Starnes A, Hamby D, Gordon N (1993) Building and evaluating family support initiative. Paul H Brookes Pub Co, Baltimore.

14. Gutstein SE (2009) Empowering families through Relationship Development Intervention: an important part of the biopsychosocial management of autism spectrum disorders. Ann Clin Psychiatry 21: 174-182.

15. Luong J, Yoder MK, Canham D (2009) Southeast Asian parents raising a child with autism: a qualitative investigation of coping styles. J Sch Nurs 25: 222-229.

16. Neely-Barnes S, Graff JC, Marcenko M, Weber L (2008) Family decision making: benefits to persons with developmental disabilities and their family members. Intellect Dev Disabil 46: 93-105.

17. Sanders MR (1999) Triple P-Positive Parenting Program: towards an empirically validated multilevel parenting and family support strategy for the prevention of behavior and emotional problems in children. Clin Child Fam Psychol Rev 2 : 71-90.

18. Sanders MR (2012) Development, evaluation, and multinational dissemination of the triple P-Positive Parenting Program. Annu Rev Clin Psychol 8: 345-379.

19. Biglan A, Flay BR, Embry DD, Sandler IN (2012) The critical role of nurturing environments for promoting human well-being. Am Psychol 67: 257-271.

20. Sanders MR (2008) Triple P-Positive Parenting Program as a public health approach to strengthening parenting. J Fam Psychol 22: 506-517.

21. de Graaf I, Speetjens P, Smit F, de Wolff M, Tavecchio L (2008) Effectiveness of the Triple $\mathrm{P}$ Positive Parenting Program on behavioral problems in children: a meta-analysis. Behav Modif 32: 714-735.

22. Nowak C, Heinrichs N (2008) A comprehensive meta-analysis of Triple P-Positive Parenting Program using hierarchical linear modeling: effectiveness and moderating variables. Clin Child Fam Psychol Rev 11: 114-144.

23. Thomas R, Zimmer-Gembeck MJ (2007) Behavioral outcomes of Parent-Child Interaction Therapy and Triple P-Positive Parenting Program: a review and meta-analysis. J Abnorm Child Psychol 35: 475-495.

24. Leung C, Fan A, Sanders MR (2013) The effectiveness of a Group Triple $P$ with Chinese parents who have a child with developmental disabilities: randomized controlled trial. Res Dev Disabil 34: 976-984.

25. Leung C, Sanders MR, Leung S, Mak R, Lau J (2003) An outcome evaluation of the implementation of the Triple P-Positive Parenting Program in Hong Kong Fam Process 42: 531-544.

26. Fujiwara T, Kato N, Sanders MR (2011) Effectiveness of Group Positive Parenting Program (Triple $P$ ) in changing child behavior, parenting style, and parental adjustment: An intervention study in Japan. J Child Fam Stud 20: 804814

27. Matsumoto Y, SofronoffK, Sanders MR (2007) The efficacy and acceptability of the Triple P-Positive Parenting Program with Japanese Parents. Behav Change 24: 205-218. 
Citation: Wakimizu R, Fujioka H, lejima A, Miyamoto S (2014) Effectiveness of the Group-Based Positive Parenting Program with Japanese Families Raising a Child with Developmental Disabilities: A Longitudinal Study. J Psychol Abnorm Child 3: 113. doi:10.4172/2329-9525.1000113

28. Matsumoto Y, Sofronoff K, Sanders MR (2010) Investigation of the effectiveness and social validity of the Triple $P$ Positive Parenting Program in Japanese society. J Fam Psychol 24: 87-91.

29. Wakimizu R, Fujioka H, Yoneyama A (2010) Empowerment process for families rearing children with developmental disorders in Japan. Nurs Health Sci 12: 322-328.

30. Goodman R (1997) The Strengths and Difficulties Questionnaire: a research note. J Child Psychol Psychiatry 38: 581-586.

31. Matsuishi T, Nagano M, Araki Y, Tanaka Y, Iwasaki M, et al. (2008) Scale properties of the Japanese version of the Strengths and Difficulties Questionnaire (SDQ): a study of infant and school children in community samples. Brain Dev 30: 410-415.

32. Smedje H, Broman JE, Hetta J, von Knorring AL (1999) Psychometric properties of a Swedish version of the "Strengths and Difficulties Questionnaire". Eur Child Adolesc Psychiatry 8: 63-70.

33. Arnold DS, O'Leary SG, Wolff LS, Acker MM (1993) The parenting scale: A measure of dysfunctional parenting in discipline situations. Psychol Assessment 5: $137-144$.

34. Lovibond PF, Lovibond SH (1995) The structure of negative emotional states: comparison of the Depression Anxiety Stress Scales (DASS) with the Beck Depression and Anxiety Inventories. Behav Res Ther 33: 335-343.

35. Turner KMT, Sanders MR, Markie-Dadds C (1999) Practitioner's manual for primary care Triple P. Families International Publishing, Brisbane, Australia.

36. Herbert RJ, Gagnon AJ, Rennick JE, O'Loughlin JL (2009) A systematic review of questionnaires measuring health-related empowerment. Res Theory Nurs Pract 23: 107-132.

37. Wakimizu R, Fujioka H, Yoneyama A, lejima A, Miyamoto S (2011) Factors associated with the empowerment of Japanese families raising a child with developmental disorders. Res Dev Disabil 32: 1030-1037.

38. Wakimizu R, Fujioka H, Furuya K, Miyamato S, lejima A, et al. (2010) Development of the Japanese version of Family Empowerment Scale (J-FES). $\mathrm{J}$ Health Welfare Stat 11: 33-41.

39. Turner KMT, Markie-Dadds C, Sanders MR (1998) Facilitator's manual for group triple P. Families International Publishing Pty Ltd., Milton, Australia.
40. Sanders MR, Markie-Dadds C, Tully LA, Bor W (2000) The triple P-positive parenting program: a comparison of enhanced, standard, and self-directed behavioral family intervention for parents of children with early onset conduct problems. J Consult Clin Psychol 68: 624-640.

41. Cohen JE (1988) Statistical Power Analysis for the Behavioral Sciences. Hillsdale, NJ: Lawrence Erlbaum Associates, Inc.

42. Ministry of Health, Labour and Welfare (2013) Annual Health, Labour and Welfare Report 2011-2012.

43. Ministry of Health, Labour and Welfare (2013) Strengths and Difficulties Questionnaire.

44. Ministry of Health, Labour and Welfare (2010) National Livelihood Survey.

45. Naganuma Y, Tachimori H, Kawakami N, Takeshima T, Ono Y, et al. (2006) Twelve-month use of mental health services in four areas in Japan: findings from the World Mental Health Japan Survey 2002-2003. Psychiatry Clin Neurosci 60: 240-248.

46. Duchovic CA, Gerkensmeyer JE, Wu J (2009) Factors associated with parental distress. J Child Adolesc Psychiatr Nurs 22: 40-48.

47. Dubowitz H, Black MM, Cox CE, Kerr MA, Litrownik AJ, et al. (2001) Father involvement and children's functioning at age 6 years: a multisite study. Child Maltreat 6: 300-309.

48. Yogman MW, Kindlon D, Earls F (1995) Father involvement and cognitive/ behavioral outcomes of preterm infants. J Am Acad Child Adolesc Psychiatry 34: 58-66.

49. McConachie $\mathrm{H}$ (1994) Implications of a model of stress and coping for services to families of young disabled children. Child Care Health Dev 20: 37-46.

50. Dempsey I, Dunst C (2004) Help giving styles and parent empowerment in families with a young child with a disability. J Intellect Dev Dis 29: 40-51.

51. Mazzucchelli TG, Sanders MR (2010) Facilitating practitioner ï $\neg$,exibility within an empirically supported intervention: Lessons from a system of parenting support. Clin Psychol-Sci Pr 17: 238-252.

52. Strauss K, Vicari S, Valeri G, D'Elia L, Arima S, et al. (2012) Parent inclusion in Early Intensive Behavioral Intervention: the influence of parental stress, paren treatment fidelity and parent-mediated generalization of behavior targets on child outcomes. Res Dev Disabil 3: 688-703. 